\title{
Magnetic-field control of the exciton quantum beats phase in InGaAs/GaAs quantum dots
}

\author{
B. Siarry, ${ }^{1}$ B. Eble, ${ }^{1, *}$ F. Bernardot, ${ }^{1, \dagger}$ P. Grinberg, ${ }^{1}$ C. Testelin, ${ }^{1}$ M. Chamarro, ${ }^{1}$ and A. Lemaître ${ }^{2}$ \\ ${ }^{1}$ Sorbonne Universités, UPMC Univ Paris 06, CNRS-UMR 7588, Institut des NanoSciences de Paris, F-75005, Paris, France \\ ${ }^{2}$ CNRS, UPR 20, Laboratoire de Photonique et Nanostructures, Route de Nozay, F-91460 Marcoussis, France
}

(Received 16 July 2015; published 28 October 2015)

\begin{abstract}
We demonstrate here the phase control of the neutral exciton quantum beats in InGaAs/GaAs quantum dots. A longitudinal magnetic field is used as a tuning parameter to change the phase of the oscillations in a deterministic way. This effect arises from the competition between the Zeeman splitting and the electron/hole exchange interaction on the exciton dipole symmetry. To explore this mechanism, we have developed a pump-probe setup based on the optical heterodyne detection of the quantum dots reflectivity allowing one to measure the exciton dynamics from a small quantum dots ensemble ( $\sim 300)$. Particular attention is paid to give a detailed theoretical analysis of the measurements. The experimental results are in excellent agreement with the model.
\end{abstract}

DOI: 10.1103/PhysRevB.92.155315

PACS number(s): 78.47.jg, 78.67.Hc, 78.55.Cr

\section{INTRODUCTION}

Semiconductor quantum dots (QDs) have revealed very promising and conclusive demonstrations of the quantum control of the exciton coherence by optical means. The possibility to drive the QD in the Rabi-flopping regime with resonant ultrashort laser pulses has been shown, yielding a precise determination of the quantum phase between the ground and excited states [1,2]. However, neutral quantum dots exhibit richer physics than a two-level system, as the exciton states present a level-degeneracy lifting induced by electron/hole anisotropic exchange interaction (few tens of $\mu \mathrm{eV}$ ); this fine structure splitting (FSS) naturally builds up a V-shaped system, offering the extra possibilities to write and read the coherent superposition of the two excited states. The resulting quantum beats associated to the FSS have been already reported, showing a coherence time lasting over few hundreds of picosecond [3-5].

Recent progresses have shown the possibility to tune the magnitude of the FSS with external parameters, providing new degrees of freedom for manipulating the quantum beats. For example, applying electric field leads to a progressive and deterministic cancellation of the FSS up to its inversion [6]. Boyer de la Giroday et al. have used this FSS electric-field tuning control in order to manipulate the quantum beats phase at subnanosecond time scale with electric pulses of duration shorter than the coherence time [7]. In this way, they achieved a phase-shift operation close to unit fidelity, provided the native FSS magnitude is small enough to increase the electric-field tuning sensitivity.

Resonant or quasiresonant excitation allows one to transfer an optical coherence to the $\mathrm{V}$-shaped system with respect to the optical selection rules. The two exciton states denoted $|X\rangle$ and $|Y\rangle$ are related to optical dipoles mainly aligned respectively

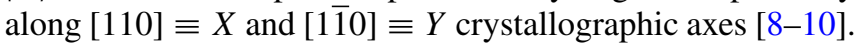
As a consequence, a resonant optical field with a polarization having both $X$ and $Y$ components imprints a determinate initial phase in between the $|X\rangle$ and $|Y\rangle$ states [11].

\footnotetext{
*benoit.eble@insp.jussieu.fr

†Also working at Université Paris Diderot, Sorbonne Paris Cité, F-75013 Paris, France.
}

We propose here a more sophisticated experimental protocol allowing the quantum-beat phase manipulation, not only by means of light polarization control, but also by applying a longitudinal magnetic field. The key role of the magnetic field consists in changing the symmetry of the linear dipoles into elliptic ones (see Fig. 1): the optical selection rules evolution results in a competition between the longitudinal magnetic field and the effective in-plane electron/hole exchange field, providing a magnetic field tuning of the exciton quantum beats phase. At this point, it is worth noting that an Overhauser field aligned with the [001] direction, coming from a significant nuclear spin polarization inside the quantum dot, can play a similar role to the applied magnetic field we mentioned above [12-14].

We present here an experimental study confirming the magnetic-field tuning of the quantum-beats phase. A pump/probe setup is employed where the reflectivity is measured by optical heterodyne detection allowing one simultaneously to increase the detection of photoinduced signal, and to perform various experimental polarization configurations. The paper is organized as follows. We will present in Sec. II the sample and the experimental pump-probe heterodyne setup; then Sec. III is devoted to the theoretical analysis of the experiments. Finally, the last section will present the experimental results, in good agreement with the theoretical model.

\section{SAMPLE AND EXPERIMENTAL SETUP}

The studied sample contains three layers of neutral InGaAs/GaAs QDs, with a nominal density of $\sim 5 \times 10^{10} \mathrm{~cm}^{-2}$. The QDs were embedded inside a $\lambda$-planar GaAs cavity made of $24 \mathrm{AlGaAs} / \mathrm{GaAs}$ Bragg pairs for the bottom mirror and 12 for the top mirror. The role of the cavity is to increase the detection sensitivity of the signal [15] and its $Q$ factor is close to $\sim 2000$.

To measure the exciton dynamics, we use a reflectiontype pump-probe setup, as depicted in Fig. 2. A picosecond mode-locked Ti:Sa laser is split into three beams (pump, probe, and reference beams) with energy matching the optical mode of the cavity centered at $1.337 \mathrm{eV}$. The pump and probe beams are focused on the sample with a microscope objective (N.A. $=0.5)$ giving a spot diameter of $\sim 1 \mu \mathrm{m}$. In 


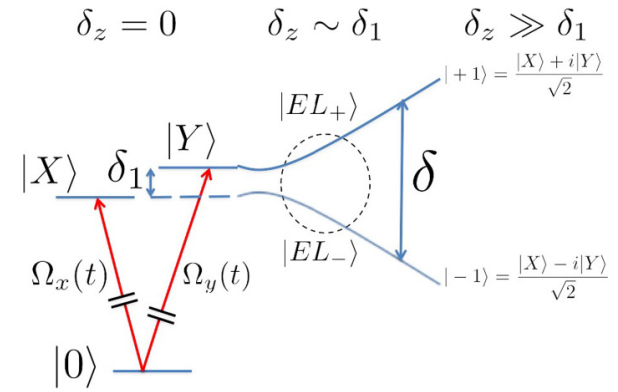

FIG. 1. (Color online) Scheme of the V-shape system, as a function of the Zeeman splitting $\delta_{z}$. When $\delta_{z}=0$, the optical dipoles are linearly polarized and parallel respectively to the $X$ and $Y$ axes. Their energies are split by the FSS $\delta_{1}$. In the limit $\delta_{z} \gg \delta_{1}$, the optical dipoles become circularly polarized, whereas in the intermediate regime defined by $\delta_{z} \sim \delta_{1}$ and delimited by the dashed circle, the dipoles show elliptic symmetry with principle axes still aligned with $X$ and $Y$ directions. Their energy separation simply writes $\delta=\sqrt{\delta_{1}^{2}+\delta_{z}^{2}} . \Omega_{x}(t)$ and $\Omega_{y}(t)$ denote the optical pulse couplings with respect to the excitons dipole symmetry at zero magnetic field.

this way, we estimate that only $N_{\mathrm{QD}} \sim 300$ QDs spectrally in resonance with the cavity mode contribute to the reflectivity signal. The standard deviation of the probed QDs sample $\sqrt{N_{\mathrm{QD}}} / N_{\mathrm{QD}} \simeq 5 \%$ is small enough to catch the essential aspects of the exciton dynamics with average values [16]. New experimental strategies are required to increase the signal-to-noise ratio of the small number of probed QDs. In this sense, we developed an optical heterodyne amplification of the reflectivity signal $[17,18]$. The probe and reference beams are passed through acousto-optic modulators leading to an optical frequency shift of the first diffraction order: the probe optical frequency becomes $\omega_{p r}=\omega_{L}+\omega_{1}$ and the reference $\omega_{\text {ref }}=\omega_{L}+\omega_{2}$, with $\omega_{1}=110 \mathrm{MHz}$ and $\omega_{2}=112 \mathrm{MHz}$. We note here that $\omega_{1}, \omega_{2}$ are several orders of magnitude less than the cavity-mode linewidth. Then, the reflected part of the probe pulse having interacted with the QDs and the reference beam are temporally overlapped onto the avalanche

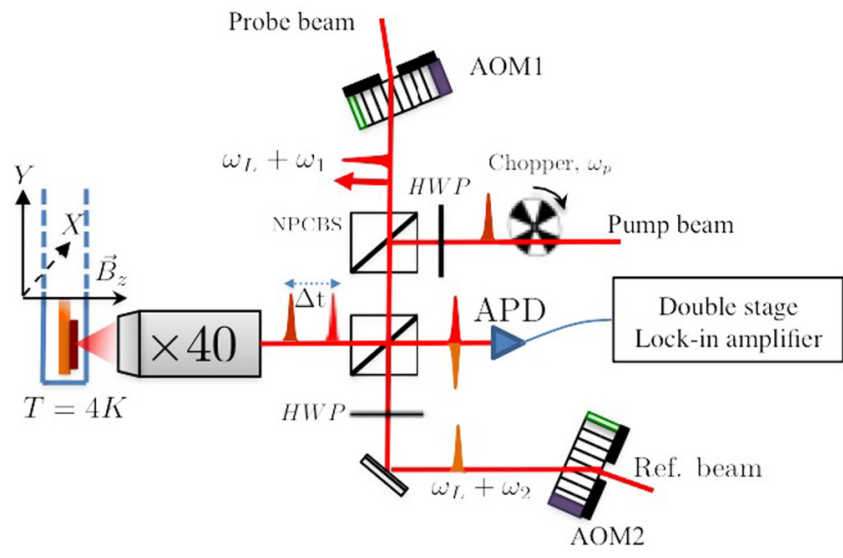

FIG. 2. (Color online) Scheme of the pump/probe setup with heterodyne amplification of the reflectivity signal. AOM: acoustooptics modulator; HWP: half-wave plate; NPCBS: nonpolarizing cube beam splitter; APD: avalanche photodiode; $\Delta t$ : pump-probe delay.

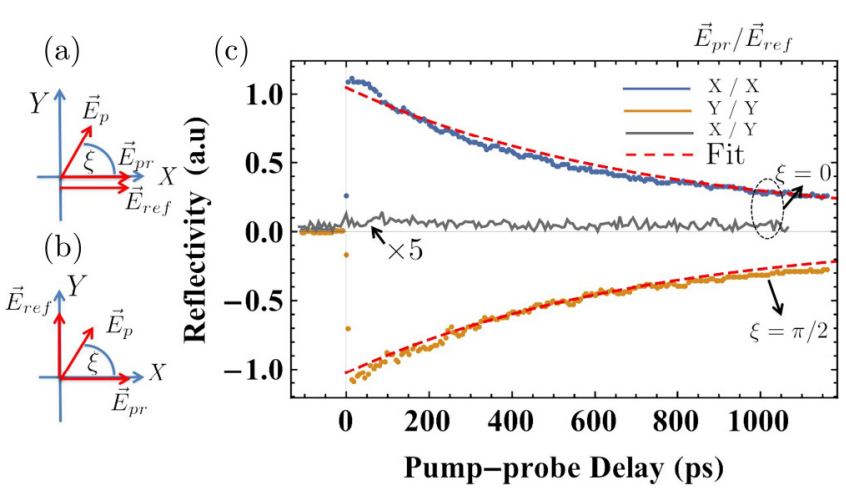

FIG. 3. (Color online) Description of the experimental polarization configurations. (a) The linear polarization of the probe and reference fields are parallel and aligned with the crystallographic axes $X$, whereas they are set perpendicular in (b). (c) Dynamics of the reflectivity signal: the pump polarization is parallel to an eigenstate direction $X$ or $Y$, and the polarization of the probe and reference are specified in the legend. The signal detected with orthogonal reference and probe polarizations has been multiplied by five.

photodiode, giving rise to the two-wave mixing signal, i.e., the heterodyne signal beating at $\left|\omega_{1}-\omega_{2}\right|=2 \mathrm{MHz}$ in our case.

To go further in describing the advantage of the heterodyne detection, we can express the whole intensity contributions received by the APD:

$$
\begin{aligned}
&\left\langle\left(\vec{E}_{p, r}(t) e^{i \omega_{L} t}+\vec{E}_{\mathrm{ref}}(t) e^{i\left(\omega_{L}+\omega_{2}\right) t}+\left(\vec{E}_{p r, r}(t)\right.\right.\right. \\
&\left.\left.\left.\quad+\vec{E}^{\mathrm{QD}}(t)\right) e^{i\left(\omega_{L}+\omega_{1}\right) t}\right) \times \mathrm{c} . \mathrm{c} .\right\rangle \\
& \simeq\left\langle\vec{E}_{p, r}^{2}\right\rangle+\left\langle\vec{E}_{\mathrm{ref}}^{2}\right\rangle+2\left\langle\left(\vec{E}_{p r, r}+\vec{E}^{\mathrm{QD}}\right) \cdot \vec{E}_{\mathrm{ref}}\right\rangle \\
& \quad \times \cos \left(\omega_{1}-\omega_{2}\right) t,
\end{aligned}
$$

where $\vec{E}_{p, r}(t), \vec{E}_{\text {ref }}(t)$ are the electric field associated respectively to the reflected pump and reference pulse envelopes. In the same way, $\vec{E}^{\mathrm{QD}}(t)$ refers to the envelope of the probe pulse field reflected by the QDs, of which exact derivation is given further, and $\vec{E}_{p r, r}$ stands as the inherent reflected probe component that has not interacted with the QDs. $\langle\cdots\rangle$ finally denotes the detector time averaging. As an optical chopper modulates the pump intensity at a frequency $\omega_{p}=1.7 \mathrm{kHz}$, a double stage lock-in amplifier is then able to isolate $\left|\left\langle\left(\vec{E}_{p r, r}+\vec{E}^{\mathrm{QD}}\right) \cdot \vec{E}_{\text {ref }}\right\rangle\right|$ with a high pump-signal rejection, where $\vec{E}_{\text {ref }}$ plays both the role of an amplification and a polarization analysis of the QDs reflectivity signal. The average probe power is set at $0.1 \mu \mathrm{W}$, whereas the reference and pump powers are close to $5 \mu \mathrm{W}$.

The polarization configurations used in this work are described in Figs. 3(a) and 3(b) for the three beams: the pump linear polarization makes an angle $\xi$ with the $X$ axes, while the probe polarization is aligned with one of the eigenstate directions and the reference could be either parallel or perpendicular to the probe polarization direction.

Figure 3(c) provides a first insight into the experimental results. This preliminary experiment consists in measuring the decay of the reflectivity signal associated to both $|X\rangle$ and $|Y\rangle$ exciton eigenstates, which are addressed selectively by an $X$ (i.e., $\xi=0$ ) and $Y$ (i.e., $\xi=\pi / 2$ ) linearly polarized pump. In the same way, the population dynamics of the $|X\rangle(|Y\rangle)$ 
exciton is recorded with the corresponding probe polarization along $X(Y)$, and finally the reference is set parallel to the probe in order to amplify the probe reflectivity change. The heterodyne signals are shown in Fig. 3(c) and the dynamics for the $|Y\rangle$ state has been artificially set negative for clarity. Remarkably both dynamics present almost the same amplitude and are well fitted with the same monoexponential decay of $710 \pm 10 \mathrm{ps}$, giving with good approximation the radiative lifetime, assuming the exciton relaxation rate is negligible over the radiative time scale as already confirmed in Refs. [19,20]. From this experimental result, we conclude that the two optical exciton transitions have nearly the same oscillator strength; this assumption will deeply simplify the derivation of the reflected probe pulse onto the QDs in the following section.

Lastly, to verify that the polarization configurations mentioned here permit one to selectively study the exciton eigenstate population dynamics, the reference linear polarization is now rotated by $\pi / 2$ and set perpendicular to the probe. The heterodyne signal is shown by the continuous gray line in Fig. 3(c) and appears to be vanishingly small, confirming that the reflected probe acquires no significant orthogonal polarization, when the probe polarization is aligned with one of the exciton eigenstate directions.

\section{MODELIZATION OF THE PHOTOINDUCED HETERODYNE SIGNAL}

This pump-probe experiment offers a lot of possible polarization configurations giving each different aspect of the exciton dynamics, as three independent beams are involved. Before presenting a complete experimental study, this section is devoted to modelizing the photoinduced heterodyne signal, in various polarization configurations. First, we will pay attention to writing the exciton eigenstates basis in the presence of a longitudinal magnetic field. Then, the reflected probe pulsed $\vec{E}^{\mathrm{QD}}(t)$ will be calculated, and its mixing with the reference having different linear polarization direction and giving rise to the optical heterodyne beats will be discussed.

\section{A. Exciton eigenstates symmetry in the presence of a longitudinal magnetic field}

When applying a magnetic field along the growth direction, the Hamiltonian describing the exciton states is written as [21]

$$
\begin{aligned}
\hat{H}= & \left(\hbar \omega_{0}-\frac{\delta_{1}}{2}\right)|X\rangle\left\langle X\left|+\left(\hbar \omega_{0}+\frac{\delta_{1}}{2}\right)\right| Y\right\rangle\langle Y| \\
& +\frac{\delta_{z}}{2}(|+1\rangle\langle+1|-|-1\rangle\langle-1|),
\end{aligned}
$$

where $| \pm 1\rangle$ are written in Fig. $1, \delta_{1}$ is the FSS value, and $\delta_{z}$ is the Zeeman splitting given by $g_{z} \mu_{B} B_{z}$ with $g_{z}$ being the exciton longitudinal $g$ factor. Diagonalization of the Hamiltonian leads to new eigenstates denoted $\left|E L_{+}\right\rangle$and $\left|E L_{-}\right\rangle$, with their corresponding energies:

$$
\begin{aligned}
& \left|E L_{+}\right\rangle=\sin \alpha|X\rangle+i \cos \alpha|Y\rangle, \quad E_{+}=\hbar \omega_{0}+\frac{1}{2} \sqrt{\delta_{1}^{2}+\delta_{z}^{2}}, \\
& \left|E L_{-}\right\rangle=\cos \alpha|X\rangle-i \sin \alpha|Y\rangle, \quad E_{-}=\hbar \omega_{0}-\frac{1}{2} \sqrt{\delta_{1}^{2}+\delta_{z}^{2}} .
\end{aligned}
$$

$\alpha$ is the angle defined as $\frac{1}{2} \arctan \left(\delta_{z} / \delta_{1}\right)$. When the condition $\delta_{z} \gg \delta_{1}$ is fulfilled, $\alpha$ tends to $\pi / 4$ and the circular symmetry of the exciton states is restored. It is now clear that for the intermediate regime of Zeeman splitting, the states present an elliptic symmetry.

When regarding more precisely the interaction with the resonant linearly polarized pump pulse, the Hamiltonian is given by

$$
\begin{aligned}
\hat{H}_{p}(t)= & -\hbar \Omega_{p}(t) e^{-i \omega_{L} t}[\cos \xi \cos \alpha+i \sin \xi \sin \alpha] \hat{\sigma}_{-0} \\
& -\hbar \Omega_{p}(t) e^{-i \omega_{L} t}[\cos \xi \sin \alpha-i \sin \xi \cos \alpha] \hat{\sigma}_{+0} \\
& + \text { H.c. }
\end{aligned}
$$

where $\hat{\sigma}_{ \pm 0}=\left|E L_{ \pm}\right\rangle\langle 0|$ and [22] $\Omega_{p}(t)=\frac{\mu_{x} E_{p}(t)}{\hbar}=\frac{\mu_{y} E_{p}(t)}{\hbar}$.

The optical coupling coefficients in $\hat{H}_{p}(t)$ expressed in the $\left\{|0\rangle,\left|E L_{-}\right\rangle,\left|E L_{+}\right\rangle\right\}$basis are complex functions of $(\xi$, $\alpha$ ), and differ for each optical transition $|0\rangle \rightarrow\left|E L_{+}\right\rangle$and $|0\rangle \rightarrow\left|E L_{-}\right\rangle$. The key point is that their arguments are different, yielding the possibility to control the initial phase (i.e., just after the passage of the pump pulse) between $\left|E L_{+}\right\rangle$ and $\left|E L_{-}\right\rangle$by changing $\alpha$ (i.e., the magnetic field) when fixing $\xi$. At this stage, it is easy to check on Eq. (2) that, for $\xi=0$ or $\xi=\pi / 2$, both optical couplings have the same arguments and are simultaneously either purely imaginary or real, preventing the phase manipulation by applying the magnetic field.

The more intriguing case is for $\xi=\pi / 4$. When this pump polarization configuration is employed, the argument of the optical coupling to $\left|E L_{+}\right\rangle$state, $\operatorname{Arg}[\sin \alpha-i \cos \alpha]$, is $\alpha-$ $\pi / 2$, whereas the other one, $\operatorname{Arg}[\cos \alpha+i \sin \alpha]$, is $\alpha$, so that the difference of both arguments becomes $\alpha$ independent. This also means that the $\xi=\pi / 4$ configuration renders inoperative the magnetic field control of the exciton quantum beats phase. From this very preliminary analysis, we conclude that lowsymmetry pump polarization direction $\xi$ is required to validate the magnetic field induced phase control protocol (i.e., for $\xi$ different from $0, \pi / 4$, and $\pi / 2$ ).

All these aspects which lie at the heart of this publication will be specified in the following and illustrated experimentally.

\section{B. Expressions of the reflectivity signals}

We propose here to express the probe electric field $\vec{E}^{\mathrm{QD}}(t)$ reflected by the QDs; this latter should be a function of $\xi, \alpha$, and the pump-probe delay $\Delta t$ but also depends on the probe polarization, while the heterodyne signal depends in addition on the reference polarization. First we consider the density operator evolution given by the Liouville equation:

$$
\frac{d \hat{\rho}}{d t}=-\frac{i}{\hbar}[\hat{H}, \hat{\rho}(t)]-\left(\frac{\partial \hat{\rho}}{\partial t}\right)_{\mathrm{rad}}-\left(\frac{\partial \hat{\rho}}{\partial t}\right)_{\mathrm{decoh}} .
$$

We use the eingenstates basis $\left\{|0\rangle,\left|E L_{-}\right\rangle,\left|E L_{+}\right\rangle\right\}$. Before the pulse arrival, the density operator is written as $\hat{\rho}\left(0^{-}\right)=\operatorname{Diag}[1,0,0]$ and becomes after pump pulse $\hat{\rho}\left(0^{+}\right)=$ $W(\infty) \hat{\rho}\left(0^{-}\right) W^{\dagger}(\infty)$, where the unitary pump operator [23] is $W(t)=e^{\frac{i}{\hbar} \int_{-\infty}^{t} \hat{H}_{p}\left(t^{\prime}\right) d t^{\prime}}$ and $\hat{H}_{p}(t)$ exists in the interval of the pump pulse duration $\tau_{p} \sim 2$ ps. The corresponding spectral width of the laser $1 / \tau_{p}$ is on the one hand much larger than the exciton splitting energy, and on the other hand comparable with 
the cavity-mode linewidth. $\hat{\rho}\left(0^{+}\right)$naturally serves as the initial condition to solve Eq. (3). Then we find for the population terms

$$
\left\{\begin{array}{l}
\rho_{00}(t)=1-e^{-t / \tau_{r}} \sin ^{2} \theta / 2, \\
\rho_{--}(t)=\frac{1}{2} e^{-t / \tau_{r}}(1+\cos 2 \alpha \cos 2 \xi) \sin ^{2} \theta / 2, \\
\rho_{++}(t)=\frac{1}{2} e^{-t / \tau_{r}}(1-\cos 2 \alpha \cos 2 \xi) \sin ^{2} \theta / 2,
\end{array}\right.
$$

and for the coherence terms of interest

$\left\{\begin{array}{l}\rho_{+-}(t)=\frac{1}{2} e^{-i\left(\frac{\delta}{\hbar}-i \gamma\right) t}[\cos 2 \xi \sin 2 \alpha-i \sin 2 \xi] \sin ^{2} \theta / 2, \\ \rho_{-+}(t)=\rho_{+-}^{*}(t)\end{array}\right.$

where $\theta=\int \Omega_{p}(t) d t$ is the Rabi area of the pump pulse, $\gamma=$ $1 / \tau_{r}+1 / T_{2}, T_{2}$ is the coherence time of the excitons beatings, and $\delta=\sqrt{\delta_{1}^{2}+\delta_{z}^{2}}$.

While $\hat{\rho}(t)$ describes the free evolution of the system after the pump pulse, we note $\hat{\sigma}(t)$ the density matrix taking into account the interaction between the QDs and the probe field for a given pump-probe delay $\Delta t$. As the pulse duration $\tau_{p}$ is much shorter than the interband coherence time [24], $\hat{\sigma}(t)$ is governed by the coherent evolution equation:

$$
\frac{d \hat{\sigma}}{d t}=-\frac{i}{\hbar}\left[\hat{H}+\hat{H}_{p r}(t-\Delta t), \hat{\sigma}\right],
$$

where $\hat{H}_{p r}(t)=-\left[\hbar \Omega_{p r}(t) e^{-i \omega_{p r} t} \cos \alpha\right] \hat{\sigma}_{-0}-\left[\hbar \Omega_{p r}(t) e^{-i \omega_{p r} t}\right.$ $\sin \alpha] \hat{\sigma}_{+0}+$ H.c. for a probe polarization direction along $X$. The solution of Eq. (4) is calculated in the first order in the probe field and can be expressed by

$$
\hat{\sigma}(t) \simeq \hat{\rho}(\Delta t)-\frac{i}{\hbar} \int_{-\infty}^{t}\left[\hat{H}_{p r}\left(t^{\prime}-\Delta t\right), \hat{\sigma}\right] d t^{\prime},
$$

with the conditions $-\tau_{p} / 2 \lesssim t^{\prime}-\Delta t \lesssim \tau_{p} / 2$ and $\sigma_{i i}(\Delta t)=$ $\rho_{i i}(\Delta t)$ indicating that the probe field does not change significantly the population terms under the experimental precautions $\left|\Omega_{p r}(t)\right| \ll\left|\Omega_{p}(t)\right|$ and $\left|\Omega_{p r}(t)\right| \tau_{p} \ll 1$. Only the second term in Eq. (5) mixes the interactions with the pump and probe fields and participates to the photoinduced signal, whereas the first term solely describes the pump-induced resonant fluorescence. As the reference beam is polarized along the crystallographic axes, it is very convenient to express $\hat{\sigma}$ in the $\{|0\rangle,|X\rangle,|Y\rangle\}$ basis, by applying the change-of-basis operator $P$ according to $\hat{\sigma}^{\star}=P \cdot \hat{\sigma} \cdot P^{\dagger}$ with

$$
P=\left(\begin{array}{ccc}
1 & 0 & 0 \\
0 & \cos \alpha & i \sin \alpha \\
0 & \sin \alpha & -i \cos \alpha
\end{array}\right)
$$

Due to this operation, the coherence elements in $\hat{\sigma}^{\star}$ result in a mixing with population and coherence terms of $\hat{\sigma}$. Then the probe-induced stimulated QDs emission-or in the classical point of view, the reflected part of the probe by the QDs-is now calculated by first expressing the quantum average dipole $\left\langle d_{j}\right\rangle(t)=\mu_{j}\left[\sigma_{j 0}^{\star}(t)+\right.$ c.c. $]$ with $j=\{X, Y\}$. In the classical far-field approximations, the $\mathrm{QD}$ radiated field is proportional to the oscillating dipole, and the two orthogonal components are

$$
\begin{aligned}
E_{X}^{\mathrm{QD}}(t)= & A_{X}(t)\left\{-\left[3+\cos 2 \xi \cos ^{2} 2 \alpha\right] e^{-\Delta t / \tau_{r}}+e^{-\gamma \Delta t}\right. \\
& \times\left[\sin 2 \xi \sin \frac{\delta \Delta t}{\hbar}-\cos 2 \xi \sin 2 \alpha \cos \frac{\delta \Delta t}{\hbar}\right] \\
& \times \sin 2 \alpha\} e^{i \omega_{p r} t}+\text { c.c. }
\end{aligned}
$$

and

$$
\begin{aligned}
E_{Y}^{\mathrm{QD}}(t)= & A_{Y}(t)\left\{i \cos 2 \xi \cos 2 \alpha \sin 2 \alpha e^{-\Delta t / \tau_{r}}-e^{-\gamma \Delta t}\right. \\
& \times\left[\cos \frac{\delta \Delta t}{\hbar}(\sin 2 \xi+i \cos 2 \xi \sin 2 \alpha \cos 2 \alpha)\right. \\
& \left.\left.+\sin \frac{\delta \Delta t}{\hbar}(\cos 2 \xi \sin 2 \alpha-i \sin 2 \xi \cos 2 \alpha)\right]\right\} \\
& \times e^{i \omega_{p r} t}+\text { c.c. },
\end{aligned}
$$

with $A_{j}(t)=\mu_{0} \omega_{p r}^{2} \beta \times i \mu_{j}\left(\int_{-\infty}^{t} \Omega_{p r}\left(t^{\prime}-\Delta t\right) d t^{\prime}\right) \times \sin ^{2} \frac{\theta}{2}$, where $\mu_{0}$ is the magnetic permeability and the complex coefficient $\beta$ takes into account the cavity effect on the probe reflection, and contains the phase factor due to the wave propagation from the QDs to the photodetector. Depending on the reference polarization, the experiment is then sensitive selectively to the QD fields polarized along the $X$ or $Y$ direction. At this stage, it is easy to verify on Eq. (7) that for $(\xi=0, \alpha=0)$ (i.e., the pump parallel to the probe polarization direction along $X$ and no applied magnetic field), the $Y$ component of the QDs emitted field $E_{Y}^{\mathrm{QD}}(t)$ is zero giving no heterodyne signal when the reference polarization is along $Y$ as demonstrated in Fig. 3(c).

As a lock-in amplifier detection is used, one needs to calculate the amplitude of the harmonic beating at $\left|\omega_{1}-\omega_{2}\right|$, noted $R$. We will consider a perfect alignment of the reference and probe polarization directions with the crystallographic axes. In our measurement, the probe polarization is kept constant along $X$ and only the pump and reference polarization change, so that we write the signal $R(\Delta t, \xi, X)(R(\Delta t, \xi, Y))$ when the reference polarization is aligned with the $X(Y)$ direction. Then we find, omitting the amplitude prefactor,

$$
\begin{aligned}
R(\Delta t & t \xi, X) \\
= & \left(3+\cos 2 \xi \cos ^{2} 2 \alpha\right) e^{-\Delta t / \tau_{r}} \\
& +e^{-\gamma \Delta t}\left(\cos 2 \xi \sin 2 \alpha \cos \frac{\delta \Delta t}{\hbar}-\sin 2 \xi \sin \frac{\delta \Delta t}{\hbar}\right) \\
& \times \sin 2 \alpha
\end{aligned}
$$

and $R(\Delta t, \xi, Y)$ is of the form $\sqrt{R_{x}^{2}+R_{y}^{2}}$ with

$$
\begin{aligned}
R_{x}= & e^{-\gamma \Delta t}\left(\cos \frac{\delta \Delta t}{\hbar} \sin 2 \xi+\sin \frac{\delta \Delta t}{\hbar} \cos 2 \xi \sin 2 \alpha\right), \\
R_{y}= & \cos 2 \alpha\left\{\cos 2 \xi \sin 2 \alpha e^{-\Delta t / \tau_{r}}\right. \\
& \left.+\left[\sin \frac{\delta \Delta t}{\hbar} \sin 2 \xi-\cos \frac{\delta \Delta t}{\hbar} \cos 2 \xi \sin 2 \alpha\right] e^{-\gamma \Delta t}\right\} .
\end{aligned}
$$




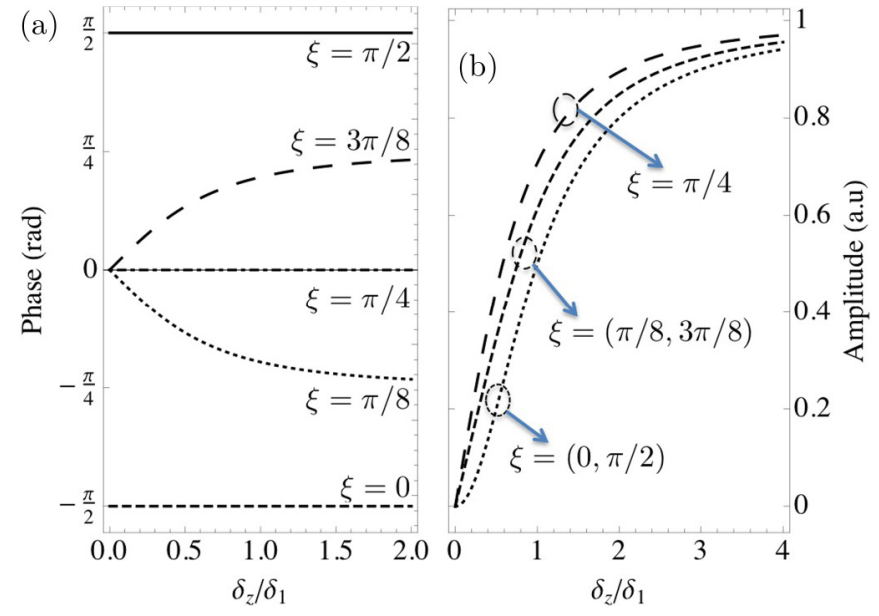

FIG. 4. (Color online) (a) Evolution of the exciton quantum beats phase with the ratio $\delta_{z} / \delta_{1}$ for different pump polarization directions $\xi$. (b) Evolution of the beats amplitude versus $\delta_{z} / \delta_{1}$ for different values of the parameter $\xi$.

At this stage of the analysis, it is important to comment more on the expression $R(\Delta t, \xi, X)$ written in (8), in which we can clearly distinguish two components: one decaying with the radiative lifetime refers to the population term, and the other one having a $\gamma$ decay corresponds to the coherent term oscillating with a period $h / \delta$. The relative amplitude of both components depends only of $(\xi, \alpha)$. Regarding more particularly the coherent term in Eq. (8), this latter can be rewritten under the form $-A(\xi, \alpha) \sin \left(\frac{\delta \Delta t}{\hbar}+\psi_{X}(\xi, \alpha)\right)$ defining a phase and an amplitude associated to the quantum beats, which are plotted respectively in Figs. 4(a) and 4(b) as a function of the ratio $\delta_{z} / \delta_{1}$ for different angles $\xi$.

Figure 4(a) confirms the qualitative arguments discussed previously in Sec. III A, where the phase remains independent of the applied magnetic field for high-symmetry pump polarization, i.e., $\xi=0, \pi / 4, \pi / 2$. However, for lowsymmetry pump polarization, the phase becomes sensitive to the magnetic field and saturates to $-\pi / 2+2 \xi$ for $0<\xi<$ $\pi / 2$ and $-3 \pi / 2+2 \xi$ for $\pi / 2<\xi<\pi$. For example, for $\xi=\pi / 8[\pi / 2](3 \pi / 8[\pi / 2])$, the phase of the quantum beats saturates to $-\pi / 4(\pi / 4)$. The amplitude related to the coherent beating shown on Fig. 4(b) increases with the magnetic field up to the saturation and looks largely independent of the angle $\xi$. When no magnetic field is applied, the coherent signal amplitude vanishes whatever the angle $\xi$ is, and only the population signal remains [see Fig. 3(c)].

To overcome this limitation, one can turn to another experimental configuration where the probe and reference polarizations are set orthogonal, giving the signal $R(\Delta t, \xi, Y)$, as the oscillating terms present in $R_{x}$ or $R_{y}$ [see Eqs. (9) and (10)] do not cancel for $\alpha=0$. However, the expression $R(\Delta t, \xi, Y)$ indicates that the recorded signal would be positive and would show a period of oscillation twice shorter than the one imposed by the sole exciton splitting energy. These observations are not corroborated by the measurements. Actually, we can show that a slight angular deviation between the probe and reference from the ideal crossed-polarization configuration as small as few tens $\mu \mathrm{rad}$ leads to a dramatic change of the signal shape $R(\Delta t, \xi, Y)$, whereas an experimental misalignment does not alter $R(\Delta t, \xi, X)$. The Appendix will provide a detailed description of this subtle effect.

\section{RESULTS AND DISCUSSION}

We present in this section the experimental results showing the reflectivity signals gathered in Fig. 5 and obtained when the probe and reference polarization directions are the same and parallel to the $X$ direction. The purpose of this work is to compare the phase of the exciton quantum beats when changing the magnitude of the longitudinal magnetic field; that is why we choose to plot the reflectivity signals in the phase domain $\Phi$ defined for each magnetic field as $\sqrt{\delta_{1}^{2}+\delta_{z}^{2}} \frac{\Delta t}{\hbar}$, rather than in the time domain $\Delta t$. Our choice renders the phase evolution more clear. This has required first to measure the FSS $\delta_{1}$ and the $g_{z}$ values.

Figure 5(a) shows the experimental results for $\xi=0$ and $\xi=\pi / 2$. When the reference polarization is set parallel to the probe one, we have demonstrated that the coherence signal is a modulation of the decaying population signal [see expression of $R(\Delta t, \xi, X)$ in Eq. (8)], which is confirmed experimentally. The period of oscillations as a function of the magnetic field can be easily fitted and finally be converted into exciton splitting energy in Fig. 5(d). Therefore, we deduce from the splitting evolution that the average values describing the probed QDs sample $\bar{\delta}_{1}$ and $\bar{g}_{z}$ are respectively [25] $15 \mu \mathrm{eV}$ and 2.8. The magnetic field value satisfying the condition $\bar{\delta}_{1}=\bar{\delta}_{z}\left(=\bar{g}_{z} \mu_{B} B_{z}\right)$ is then $B_{z} \sim 100 \mathrm{mT}$.

The continuous lines in Fig. 5(a) represent the fitting curves, which match very well the experimental data for all the magnetic field values. During the fitting procedure using the expression of $R(\Delta t, \xi, X), \tau_{r}, \overline{\delta_{1}}$, and $\bar{g}_{z}$ are maintained fixed as constant parameters, whereas only two free parameters are used: the coherence time $T_{2}$ and a global renormalization prefactor. In particular, it is remarkable that the amplitudes of both the population and coherence components which are deeply correlated, are very well reproduced simultaneously. As expected, the amplitude of the oscillating part tends to zero for $\delta_{1}>\delta_{z}$ and saturates in the regime $\delta_{1} \ll \delta_{z}$, as predicted in Fig. 4(b). Finally, the experimental results in Fig. 5(a) show in very good agreement with the modelization that the $\xi=0$ and $\xi=\pi / 2$ reflectivity signals oscillate in phase opposition whatever the magnetic field amplitude is, as demonstrated in Fig. 4(a).

Figures 5(b) and 5(c) show the reflectivity dynamics evolution with the magnetic field, for a pump polarization corresponding to $\xi=\pi / 4$ and [26] $\xi=7 \pi / 12$. Once again, as the probe and reference polarizations are set parallel, the quantum beats are in addition to the monoexponential population signal. However, this latter contribution was removed from the experimental data after the fitting procedure in Figs. 5(b) and 5(c) in order to center the oscillations and make the magnetic field dependence of the phase more clear.

Figure 5(b) shows the two main features in good agreement with the theoretical model: first, the amplitude of the oscillating component grows up when increasing the magnetic field, and secondly no phase shift is observable when changing the magnetic-field magnitude, as expected from the $\xi=\pi / 4$ experimental configuration. A dashed line joining the intersections of the curves with their respective zero-baselines centered 


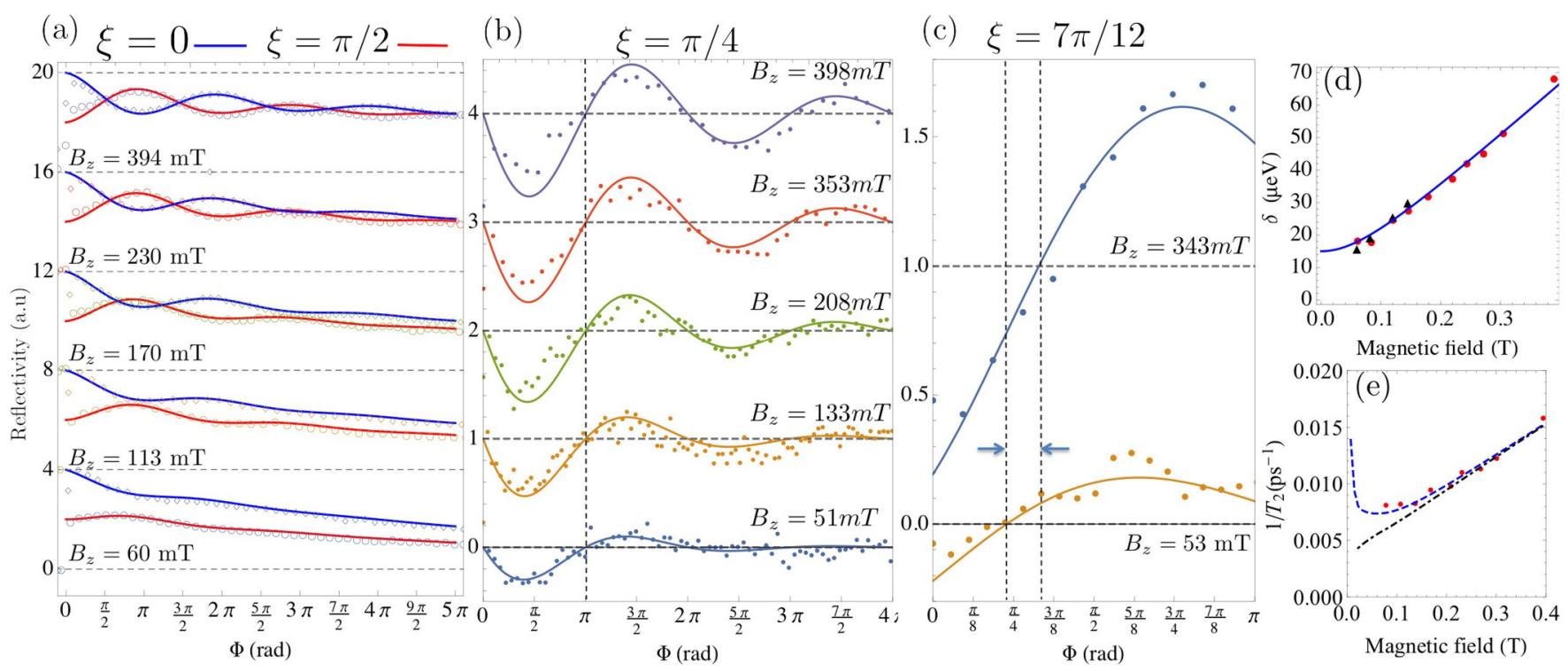

FIG. 5. (Color online) Reflectivity signals obtained when the probe and reference polarization are set parallel. (a) The pump polarization angle is $\xi=0$ and $\xi=\pi / 2$. (b) $\xi=\pi / 4$. (c) $\xi=7 \pi / 12$. (d) Evolution of the exciton splitting energy with the magnetic field, extracted from the period of oscillation. The red points come from Fig. 5(a) and $\xi=\pi / 2$, whereas the black triangles come from Fig. 7 in the Appendix. (e) Evolution of $1 / T_{2}$ as a function of the applied magnetic field. The blue dashed fitting curve takes into account the inhomogeneities of the FSS and exciton Landé- $g$ factor $g_{z}$, whereas for the black dotted curve, only the $g$-factor dispersion is considered with the following numerical values: $\Gamma_{g}=0.62$ and $T_{2}^{0}=220 \mathrm{ps}$.

at $\Phi=\pi$ appears then vertical, and is a guide for the eyes to note the absence of the magnetic-field induced phase shift.

The $\xi=7 \pi / 12$ case in Fig. 5(c) is the more interesting experimental configuration, as it differs from the high-symmetric pump polarization directions in respect of the exciton dipole polarization, and provides the evidence of the magnetic-field control of the quantum beats phase. The reflectivity signal is plotted over a half-period of oscillation (i.e., $0<\Phi<\pi$ ). We select only two data for clarity: one measured at low magnetic field $\left(B_{z}=53 \mathrm{mT}\right.$ corresponding to $\alpha_{\min } \simeq 0.26$ ), and the other one at high magnetic field $\left(B_{z}=343 \mathrm{mT}\right.$ corresponding to $\alpha_{\text {max }} \simeq 0.65$, close to its maximum limit of $\pi / 4$ ). In both cases, the theoretical model fits the data satisfactorily. For the higher magnetic field the curve intersects the zero baseline for $\Phi \simeq \pi / 3$ (see Sec. III B). A vertical line is added on the plot, as a cursor pointing at this specific angle. The second vertical line points to the zero of the second curve. It is clear that the distance between these two lines reveals the magnetic-field induced phase shift in the exciton quantum beats. The corresponding amplitude of the phase shift is simply written as $\left|\Psi_{X}\left(\alpha_{\max }, 7 \pi / 12\right)-\Psi_{X}\left(\alpha_{\min }, 7 \pi / 12\right)\right|$ and has a value of $\simeq 0.38 \mathrm{rad}$ (close to $\pi / 8$ ).

Until now, the inhomogeneities inherent to the probed QDs sample have been overlooked. In this regard, the coherence rate $1 / T_{2}$ evolution with the magnetic field gives quantitative details on the dispersion of the $\delta_{1}$ and $g_{z}$ related distributions. Figure 5(e) shows the $1 / T_{2}$ evolution as a function of the applied magnetic field, extracted from the $\xi=\pi / 2$ experiments. Very similar trends are found with other pump linear polarizations $\xi$. The dependence looks linear for the highest magnetic fields and deviates substantially from the linear behavior for $B_{z}<150 \mathrm{mT}$. These observations are then compatible with the following description: when $B_{z}>$
$150 \mathrm{mT}$, the dispersion of the exciton Landé $g$ factor dominates the quantum beats damping providing a linear dependence of $1 / T_{2}$ versus $B_{z}$, with a slope close to $\sim \mu_{B} \Gamma_{g} / 2 \hbar$, where $\Gamma_{g}$ is the FWHM of a Lorentzian distribution associated to $g_{z}$; for the lowest magnetic fields, i.e., $B_{z}<150 \mathrm{mT}$, both $\delta_{1}$ and $g_{z}$ dispersions combine. To go further quantitatively, the dispersions $\Gamma_{g}$ and $\Gamma_{\delta_{1}}$ can be simultaneously evaluated, under the reasonable assumptions of uncorrelated distributions, by averaging numerically the coherent part of the reflectivity signal over both distributions for each magnetic field, as follows:

$$
e^{-\Delta t / T_{2}^{0}} \iint \sin ^{2} 2 \alpha \cos \frac{\delta \Delta t}{\hbar} L_{\bar{\delta}_{1}, \Gamma_{\delta_{1}}}^{\left(\delta_{1}\right)} L_{\bar{g}_{z}, \Gamma_{g}}^{\left(g_{z}\right)} d g_{z} d \delta_{1},
$$

where $L_{\bar{x}, \Gamma_{x}}^{(x)}$ is the Lorentzian distribution centered on $\bar{x}$ and with a dispersion $\Gamma_{x} ; T_{2}^{0}$ is the expected coherence time at zero magnetic field. Equation (11) is then numerically approximated by $e^{-\Delta t / T_{2}^{\star}} \sin ^{2}(2 \bar{\alpha}) \cos (\bar{\delta} \Delta t / \hbar)$, where $\bar{\alpha}$ and $\bar{\delta}$ are the quantities used in the fitting procedure as constant parameters for a fixed magnetic field and evaluated with the average values $\bar{\delta}_{1}$ and $\bar{g}_{z} ; T_{2}^{\star}$ is the inhomogeneous coherence time taking into account the whole dispersed parameters. It is then possible to find the best set of parameters $T_{2}^{0}, \Gamma_{g}$, and $\Gamma_{\delta_{1}}$, which makes the $1 / T_{2}^{\star}$ dependence with the magnetic field as close as possible to the measured one. The blue dashed curve in Fig. 4(e) shows the $1 / T_{2}^{\star}$ evolution for $T_{2}^{0}=310 \mathrm{ps,}$ $\Gamma_{g}=0.62$, and $\Gamma_{\delta_{1}}=5.9 \mu \mathrm{eV}$, and presents the same trends as the experimental results. More precisely, the fast increasing of the coherence rate below $B_{z}=150 \mathrm{mT}$ is well reproduced. Then we can conclude that, for our sample, $\delta_{1}$ is closed to $15 \pm 3 \mu \mathrm{eV}$ and $g_{z}=2.8 \pm 0.31$. 


\section{CONCLUSION}

We have demonstrated the possibility to tune the exciton quantum beats phase by applying a longitudinal magnetic field. The experiments are described by the model with a very good accuracy.

The magnitude of the FSS $\delta_{1}$, as well as the pump polarization direction $\xi$ define the range where the phase becomes sensitive to the magnetic field. In our sample, $\delta_{1}$ being only $15 \mu \mathrm{eV}$, the magnetic field reaching the saturation of the phase is then restricted to $\sim 100 \mathrm{mT}$ [see Fig. 2(a)]. However, a relatively small value of the FSS, indicating moderate anisotropic QDs seems to ensure similar oscillator strength of the $|X\rangle$ and $|Y\rangle$ exciton states.

Finally, heterodyne amplification of the reflectivity should provide an efficient tool to study exciton dynamics at the level of a single quantum dot.

\section{ACKNOWLEDGMENT}

We are grateful to M. Bernard, F. Margaillan, S. Majrab, and $\mathrm{F}$. Breton for technical assistance.

\section{APPENDIX: ON THE REFLECTIVITY SIGNAL OBTAINED IN THE CROSS-POLARIZED PROBE-REFERENCE CONFIGURATION}

This Appendix is devoted to finely analyze the experimental results when the probe and reference beams are crosspolarized. We will show that the reflectivy signal can be fully understood by taking into account a nonperfect orthogonality between the probe and reference polarizations. This angular deviation amplifies an extra contribution to the heterodyne detection by mixing the reference and the reflected probe onto the sample defined as $\vec{E}_{p r, r}$ in Sec. II.

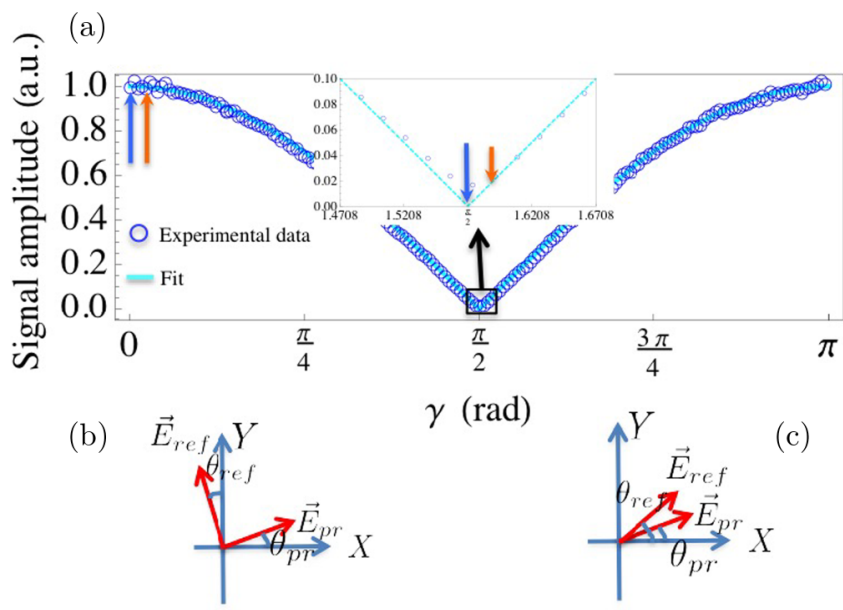

FIG. 6. (Color online) (a) Amplitude of the heterodyne signal as a function of the reference polarization direction $\gamma$, when the pump is turned off. The data is fitted by $|\cos \gamma|$. (b) Angular deviations of the reference and probe polarization used to express the reflectivity signal measured with the reference-probe cross-polarization configuration. (c) Angular deviations of the reference and probe polarization in the case of the quasicollinear configuration.

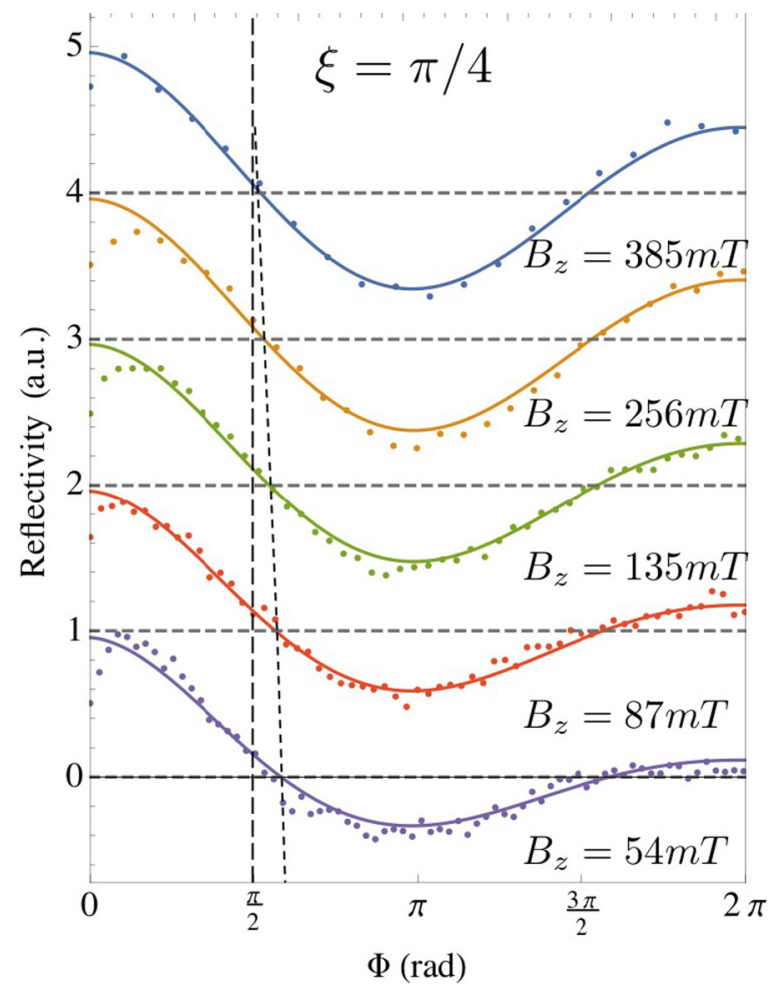

FIG. 7. (Color online) Reflectivity signals obtained when the probe and reference beams are (quasi)cross-polarized, for a pump polarization direction $\xi=\pi / 4$.

Figure 6(a) shows the heterodyne signal amplitude by rotating the reference linear polarization by an angle $\gamma$ when the probe polarization is parallel to $X$ and the pump beam is switched off. This signal analyzed at the frequency $\left|\omega_{1}-\omega_{2}\right|$ with the first stage of the lock-in amplifier,confirms that a substantial part of the probe (i.e., $\vec{E}_{p r, r}$ ) is inevitably reflected back to the photodiode. This is due, on the one hand, to the cavity mode linewidth being comparable with the pulse spectral width, and on the other hand to the focusing onto the microcavity with a high numerical aperture objective lens (N.A. $=0.5)$. Changing the experimental configuration from reference and probe beams copolarized to cross-polarized moves the operating point represented by the two blue arrows on Fig. 6(a), upon which the photoinduced signal lies on. On the other hand, the two orange arrows correspond to the real operating points relative to the two polarization configurations when considering a reference polarization angle deviation $\theta_{\text {ref }}$ to the ideal alignment [27], as depicted on Fig. 6(b). The main point is that the reference cannot distinguish along its polarization direction between the reflected probe field $\vec{E}_{p r, r}$ and the quantum dot field $\vec{E}_{B Q}$, leading to a dramatic modification of the measured reflectivity. In order to deal with the real experimental conditions, we consider also a small angular deviation noted $\theta_{p r}$ of the probe polarization with respect of the $X$ direction [see Fig. 6(b)]. We focus on the crosspolarized configuration and find that the heterodyne amplitude taking into account the whole electric fields contributions on 
the photodetector

$$
\begin{aligned}
& \mid\left\langle\left[E_{p r, r}\left(\begin{array}{c}
\cos \theta_{p r} \\
\sin \theta_{p r}
\end{array}\right) e^{i\left(\omega_{p r} t+\chi\right)}+\left(\begin{array}{c}
E_{X}^{\mathrm{QD}} \\
E_{Y}^{\mathrm{QD}}
\end{array}\right) e^{i \omega_{p r} t}\right]\right. \\
& \left.\quad \times E_{\text {ref }}^{*}\left(\begin{array}{c}
-\sin \theta_{\text {ref }} \\
\cos \theta_{\text {ref }}
\end{array}\right) e^{-i \omega_{\mathrm{ref}} t}\right\rangle .
\end{aligned}
$$

$\chi$ is the phase difference between $\vec{E}_{p r, r}$ and $\vec{E}^{\mathrm{QD}}$. Expression (A1) is then calculated with the use of reasonable assumptions: (i) $\vec{E}_{p r, r}$ keeps the same polarization as the incident probe beam; (ii) $\left|E_{p r, r} E_{\mathrm{ref}}^{*}\right| \gg\left|E_{j}^{\mathrm{QD}} E_{\mathrm{ref}}^{*}\right|$; (iii) $\left|\theta_{\mathrm{ref}}\right|$ and $\left|\theta_{p r}\right| \ll 1$.
We find then in first order in $\theta_{\text {ref }}$ and $\theta_{p r}$ :

$$
\simeq\left|E_{\mathrm{ref}}^{*}\right|\left[\left|E_{p r, r}\right||\Delta \theta|+\operatorname{Re}\left(\frac{\Delta \theta E_{p r, r} e^{i \chi} E_{Y}^{\mathrm{QD}, *}}{|\Delta \theta|\left|E_{p r, r}\right|}\right)\right],
$$

where $\Delta \theta=\theta_{\text {ref }}-\theta_{p r}$. As expected, the first term in (A2) corresponds to the change of the operating point, and carries no photoinduced signal which is present in the second term. This latter can be expressed using Eq. (7), giving the real experimental signal $R(\Delta t, \xi, Y)$ by calculating the harmonic amplitude at $\left|\omega_{1}-\omega_{2}\right|$ :

$$
\begin{aligned}
R(\Delta t, \xi, Y)= & \frac{\Delta \theta}{|\Delta \theta|}\left[\cos \chi \cos 2 \xi \sin 2 \alpha \cos 2 \alpha e^{-\Delta t / \tau_{r}}+e^{-\gamma \Delta t}\left\{(\sin \chi \sin 2 \xi-\cos \chi \cos 2 \xi \sin 2 \alpha \cos 2 \alpha) \cos \frac{\delta \Delta t}{\hbar}\right.\right. \\
& \left.\left.+(\sin \chi \cos 2 \xi \sin 2 \alpha+\cos \chi \sin 2 \xi \cos 2 \alpha) \sin \frac{\delta \Delta t}{\hbar}\right\}\right] .
\end{aligned}
$$

This expression, taking into account realistic experimental conditions, shows that the phase of the reflectivity signal, measured in the cross-polarization configuration, depends on an extra parameter $\chi$, which should be considered in the experimental data analysis. In particular, Eq. (A3) allows one to define a phase $\Psi_{Y}(\xi, \alpha, \chi)$ in the reflectivity signal. A good illustration of this is presented in Fig. 7 showing the reflectivity signal evolution with the applied magnetic field for a pump polarization direction $\xi=\pi / 4$. This figure has to be compared with Fig. 5(b). The fits using Eq. (A3) give a very good accordance with the experimental data for a single value of $\chi(0.61 \mathrm{rad})$, whatever the magnetic field is [28]. The effect of the additional parameter $\chi$ appears immediately when looking at the dashed line joining the intersection of the curves with their respective zero baselines. The line is not vertical, as opposed to the one in Fig. 5(b); this traduces the fact that $\Psi_{Y}(\xi=\pi / 4, \alpha, \chi)$ depends on the magnetic field, contrary to $\Psi_{X}(\xi=\pi / 4, \alpha)$ which does not.

Moreover, when following the same method for the copolarized configuration, and taking into account polarization misalignment [see Fig. 6(c)], the resulting reflectivity signal keeps the same form as expression (8), and the phase $\chi$ enters in the amplitude prefactor and thus plays no significant role (i.e., the small deviations $\theta_{\text {ref }}$ and $\theta_{p r}$ have no influence).

In conclusion, despite possible axis misalignment, without consequence on the physical analysis, both configurations permit one to study with accuracy the exciton phase control.
[1] T. H. Stievater, X. Li, D. G. Steel, D. Gammon, D. S. Katzer, D. Park, C. Piermarocchi, and L. J. Sham, Phys. Rev. Lett. 87, 133603 (2001).

[2] A. J. Ramsay, A. V. Gopal, E. M. Gauger, A. Nazir, B. W. Lovett, A. M. Fox, and M. S. Skolnick, Phys. Rev. Lett. 104, 017402 (2010).

[3] A. I. Tartakovskii, J. Cahill, M. N. Makhonin, D. M. Whittaker, J.-P. R. Wells, A. M. Fox, D. J. Mowbray, M. S. Skolnick, K. M. Groom, M. J. Steer, and M. Hopkinson, Phys. Rev. Lett. 93, 057401 (2004).

[4] F. Bernardot, E. Aubry, J. Tribollet, C. Testelin, M. Chamarro, L. Lombez, P.-F. Brauun, X. Marie, T. Amand, and J.-M. Gérard, Phys. Rev. B 73, 085301 (2006).

[5] A. S. Lenihan, M. V. Gurudev Dutt, D. G. Steel, S. Ghosh, and P. K. Bhattacharya, Phys. Rev. Lett. 88, 223601 (2002).

[6] K. Kowalik, O. Krebs, A. Lemaitre, B. Eble, A. Kudelski, P. Voisin, S. Seidl, and J. A. Gaj, Appl. Phys. Lett. 91, 183104 (2007).

[7] A. Boyer de la Giroday, A. J. Bennett, M. A. Pooley, R. M. Stevenson, N. Sköld, R. B. Patel, I. Farrer, D. A. Ritchie, and A. J. Shields, Phys. Rev. B 82, 241301 (2010).

[8] A. Högele, S. Seidl, M. Kroner, K. Karrai, R. J. Warburton, B. D. Gerardot, and P. M. Petroff, Phys. Rev. Lett. 93, 217401 (2004).
[9] C. Tonin, R. Hostein, V. Voliotis, R. Grousson, A. Lemaitre, and A. Martinez, Phys. Rev. B 85, 155303 (2012).

[10] I. A. Yugova, A. Greilich, E. A. Zhukov, D. R. Yakovlev, M. Bayer, D. Reuter, and A. D. Wieck, Phys. Rev. B 75, 195325 (2007).

[11] T. Flissikowski, A. Hundt, M. Lowisch, M. Rabe, and F. Henneberger, Phys. Rev. Lett. 86, 3172 (2001).

[12] T. Belhadj, C.-M. Simon, T. Amand, P. Renucci, B. Chatel, O. Krebs, A. Lemaître, P. Voisin, X. Marie, and B. Urbaszek, Phys. Rev. Lett. 103, 086601 (2009).

[13] B. Eble, O. Krebs, A. Lemaître, K. Kowalik, A. Kudelski, P. Voisin, B. Urbaszek, X. Marie, and T. Amand, Phys. Rev. B 74, 081306 (2006).

[14] A. J. Bennett, M. A. Pooley, R. M. Stevenson, I. Farrer, D. A. Ritchie, and A. J. Shields, Phys. Rev. B 84, 195401 (2011).

[15] Y. Q. Li, D. W. Steuerman, J. Berezovsky, D. S. Seferos, G. C. Bazan, and D. D. Awschalom, Appl. Phys. Lett. 88, 193126 (2006).

[16] F. Fras, B. Eble, P. Desfonds, F. Bernardot, C. Testelin, M. Chamarro, A. Miard, and A. Lemaître, Phys. Rev. B 86, 045306 (2012).

[17] A. Mecozzi and J. Mork, J. Opt. Soc. Am. B 13, 2437 (1996). 
[18] Y. Mitsumori, Y. Miyahara, K. Uedaira, H. Kosaka, S. Shimomura, S. Hiyamizu, and K. Edamatsu, Jpn. J. Appl. Phys. 50, 095004 (2011).

[19] M. Paillard, X. Marie, P. Renucci, T. Amand, A. Jbeli, and J. M. Gérard, Phys. Rev. Lett. 86, 1634 (2001).

[20] K. Kowalik, O. Krebs, A. Lemaître, J. A. Gaj, and P. Voisin, Phys. Rev. B 77, 161305(R) (2008).

[21] M. Bayer, G. Ortner, O. Stern, A. Kuther, A. A. Gorbunov, A. Forchel, P. Hawrylak, S. Fafard, K. Hinzer, T. L. Reinecke, S. N. Walck, J. P. Reithmaier, F. Klopf, and F. Schäfer, Phys. Rev. B 65, 195315 (2002).

[22] We have shown in Sec. II that the oscillator strengths are the same, implying that their related dipoles are equal, i.e., $\mu_{x}=\mu_{y}$. We also make implicit the reasonable assumption that a modest magnetic field does not change the optical dipole magnitude.

[23] F. Fras, B. Eble, B. Siarry, F. Bernardot, A. Miard, A. Lemaître, C. Testelin, and M. Chamarro, Phys. Rev. B 86, 161303 (2012).
[24] P. Borri, W. Langbein, S. Schneider, U. Woggon, R. L. Sellin, D. Ouyang, and D. Bimberg, Phys. Rev. Lett. 87, 157401 (2001).

[25] M. Sénès, B. Urbaszek, X. Marie, T. Amand, J. Tribollet, F. Bernardot, C. Testelin, M. Chamarro, and J.-M. Gérard, Phys. Rev. B 71, 115334 (2005).

[26] The experimental configuration $\xi=7 \pi / 12$ was chosen to get closer to $\xi=\pi / 8[\pi / 2]$ without compensating the native birefringence of the double cube beam splitters system (see Fig. 2), which was the case for $\xi=\pi / 4$.

[27] The deviation of the operating point associated to nonzero $\theta_{\text {ref }}$ has been deliberately overestimated on Fig. 6(b) for clarity.

[28] We have performed other experimental runs with different pump polarization directions $\xi$ (not shown here) in the cross-polarized probe and reference configuration. It appears clearly that, when fitting the experimental data for various magnetic fields, a unique value of $\chi$ is needed. However, the $\chi$ value depends slightly on the experimental conditions, and especially on the focusing onto the sample. 